Dossiê: Segurança pública e reforma das polícias na América Latina

\title{
Confianza en la policía en Chile Un arma de doble filo
}

\author{
Trust in the police in Chile \\ A double-edged sword
}

Lucía Dammert*

\begin{abstract}
Resumen: Carabineros es una de las policías mejor evaluadas en América Latina. Una de sus características es la positiva evaluación ciudadana que la instala como la institución con mayor nivel de confianza. Sin embargo este elemento también ha limitado la intervención política sobre los procesos institucionales e inhibido las reformas que emanan de actores sociales y políticos en áreas que requieren ser revisadas. El artículo describe el proceso chileno marcado por niveles de criminalidad estables, creciente temor, alta preocupación por el delito y baja evaluación de los actores del sistema. Paradojalmente, la institución policial parece no ser afectada por el panorama de molestia y preocupación ciudadana, por el contrario es calificada positivamente. También el documento muestra que el indicador de confianza debe ser analizado con mayor detalle y focalizar en aquellos grupos que muestran más desconfianza e insatisfacción en el trabajo policial: pobres, jóvenes y aquellos que han tenido contacto con funcionarios policiales.
\end{abstract}

Palabras clave: Reforma policial. Confianza. Carabineros. Chile.

Abstract: Carabineros is one of the best evaluated police institutions in Latin America. One of its features is positive citizen assessment with the highest level of trust. However, this element has also limited political intervention on institutional processes and inhibited reforms emanating from social and political actors in areas that need to be reviewed. The article describes the Chilean process marked by low and stable levels of crime, growing fear, concern over high crime and negative evaluation of criminal justice actors. Paradoxically, the police institution seems not to be affected by the panorama of annoyance and public concern. On the contrary, it is rated positively. The text also argues that trust should be analyzed in greater detail and focused on those groups that show more distrust and dissatisfaction in police work: the poor, young people and those who have had contact with police officers.

Keywords: Police reform. Trust. Carabineros. Chile.

\footnotetext{
* Doctora en Ciencia Política de la Universidad de Leiden (Holanda), profesor asociado de la Facultad de Humanidades de la Universidad de Santiago de Chile, Chile <lucia.dammert@, usach.cl>.
}

Civitas, Porto Alegre, v. 16, n. 4, p. 575-594, out.-dez. 2016

Exceto onde especificado diferentemente, a matéria publicada neste periódico 


\section{Introducción}

La reforma de la policía es una tarea pendiente en América Latina. Si bien muchos países de la región han enfrentado procesos de cambio institucional en las últimas décadas los resultados no han sido claros. El aumento del delito y la violencia ha demostrado que las instituciones estatales no logran (o no quieren) enfrentarla con la rapidez necesaria. Chile siempre ha sido considerado una excepción en este gris panorama. Los estudios sobre la policía latinoamericana permanentemente han enfatizado la diferencia del caso chileno especialmente vinculado con la presencia de una policía efectiva, donde la corrupción no es un problema cotidiano y con altos niveles de profesionalización.

La literatura sobre los cambios estructurales necesarios para avanzar hacia la construcción de una policía democrática muchas veces evita revisar los casos considerados exitosos o prometedores (Pliscoff, 2008). El presente artículo busca colocar a Chile en el marco de análisis de las fallidas y complejas reformas policiales implementadas desde el regreso de la democracia. El rol de la confianza ciudadana es evaluado como un elemento que puede inhibir el poder político impulsor de reformas evidentes o de un mayor control civil sobre las políticas de seguridad pública.

La agenda de cambios en las instituciones policiales en Chile es amplia. Desde retirar los quórums constitucionales requeridos para cambiar la Ley Orgánica de Carabineros de Chile hasta incluir profesores civiles o sugerir nuevos contenidos en los sistemas de información policial. Por supuesto que aumentar los niveles de transparencia de la información es clave. En la actualidad un porcentaje importante de los requerimientos de información por medio de la Ley de Transparencia son rechazados por las instituciones aduciendo situaciones de seguridad nacional y falta de personal dedicado a estas tareas. ${ }^{1}$ También es secreta la distribución del personal policial en el territorio.

En Chile existen dos instituciones policiales: Carabineros encargada principalmente de la prevención y el control del delito y la Policía de Investigaciones dedicada a la investigación de los delitos. Si bien esta división de tareas es clara en la arquitectura jurídica del país, en la actualidad debido Carabineros desarrolla también tareas investigativas.

El articulo describe la situación de inseguridad y crimen en Chile en las últimas décadas en su primera sección. Posteriormente se analiza la institución policial y la relación con la ciudadanía marcada por altos niveles

\footnotetext{
${ }^{1}$ Para un ejemplo de los casos de solicitud de información ver < consejotransparencia.cl/data casos/ftp_casos/C454-11/C454-11_decision_web.pdf> (27 nov. 16).
} 
de confianza y una generalizada percepción de profesionalismo. La tercera sección analiza algunos de los cambios desarrollados en las políticas de seguridad implementadas en las últimas décadas así como las iniciativas de política implementadas por los gobiernos que en general tienden a aumentar la dotación, la inversión y fortalecer las capacidades de actuación policiales.

\section{Crimen e inseguridad}

La violencia como problemática no es nueva en Chile. Son diversos los autores que plantean que la sociedad chilena presenta claros rasgos de represión y autoritarismo que pueden explicar la utilización de la violencia en diversos ámbitos y actividades. Debido a su carácter multifacético y dinámico, la violencia presenta características especiales en cada periodo histórico. Así por ejemplo, durante las décadas de los años 70 s y 80 s la presencia de violencia política y durante los años 90 s es la violencia criminal la que se instala en el vértice de la atención pública. En Chile, su utilización cotidiana se muestra en los altos niveles de violencia contra las mujeres y aquella que ocurre en los espacios privados así como en el aumento de los problemas de convivencia ciudadana que son resueltos generalmente mediante el uso de violencia. Así por ejemplo, diversos estudios corroboran que los niveles de violencia doméstica son incluso superiores a los encontrados en estudios similares en otros países de la región (León et al., 2014).

La preocupación por la delincuencia ha variado sistemáticamente en las últimas décadas pero se ha mantenido con un rol importante para la ciudadanía (Ramos y Guzman, 2000; Dammert y Malone, 2003). Como se puede observar en el gráfico siguiente en los últimos 16 años la delincuencia ha estado en el primer lugar de preocupación de los ciudadanos intercambiando en algunas ocasiones puntuales con la salud o la educación.

Debido a las falencias, de sistematización y homologación de información a partir de 1999 el Ministerio del Interior desarrolló un sistema de homologación de las denuncias de ambas instituciones policiales y se hizo cargo de su publicación y análisis. En la actualidad la información oficial no presenta el total de delitos denunciados sino más bien se centra en los llamados Delitos de Mayor Connotación Social (DMCS) que incluyen aquellos que el gobierno define que tienen mayor impacto público y relevancia dentro del accionar criminal. Los DMCS son: robo con violencia, robo con fuerza, robo con intimidación, robo por sorpresa hurto, lesiones, homicidio y violación. $\mathrm{Si}$ bien esta definición ha permitido focalizar los esfuerzos públicos, también ha generado cierta invisibilidad de otros hechos delictuales como los llamados delitos de cuello blanco o económicos. 
Gráfico 1. Preocupación por la delincuencia 2000-2016

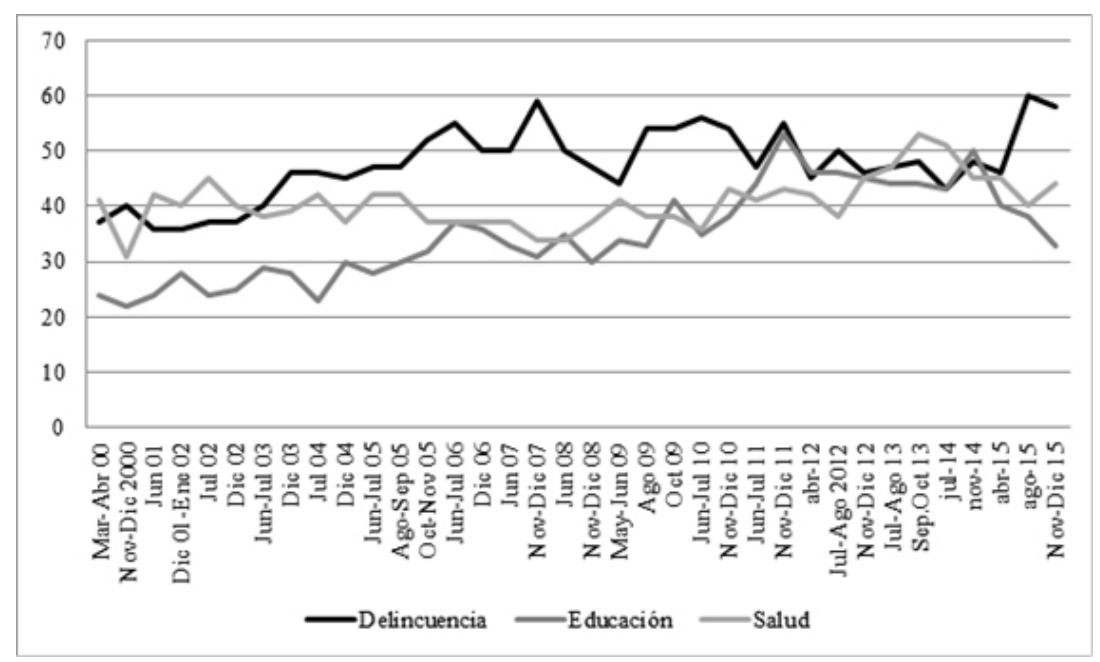

Fuente: Elaboración propia en base a encuestas CEP (2016) en base a la pregunta: Mencione los principales problemas que el gobierno debería dedicar el mayor esfuerzo a solucionar.

La información oficial de los delitos denunciados (Ministerio del Interior, 2016) y los estudios en la temática permiten resaltar varios fenómenos. En el periodo 2005-2015 la tendencia creciente de los delitos denunciados, que si bien han bajado respecto al año 2011, la información del año 2015 reconoce más de 610 mil denuncias realizadas durante el año.

El análisis de las denuncias registradas y sistematizadas por los organismos públicos permite tener un acercamiento inicial respecto del fenómeno criminal en un determinado territorio. Sin embargo, no es posible identificar la cifra negra (es decir aquellos delitos no denunciados), y por lo tanto limita el análisis sólo a aquellos delitos que son denunciados a las policías. Es por esto que las encuestas de victimización aparecen como una herramienta fundamental para caracterizar el fenómeno delictual. Los resultados de las encuestas nacionales de seguridad ciudadana (Enusc) realizadas en los últimos años por el gobierno evidencian una tendencia decreciente de la victimización, partiendo con niveles superiores al 38\% en el año 2005 y terminando la serie con $25 \%$ de los entrevistados habiendo sido víctimas de algún delito en el último año en la encuesta del 2014. 
Gráfico 2. Delitos denunciados (DMCS) 2005-2015

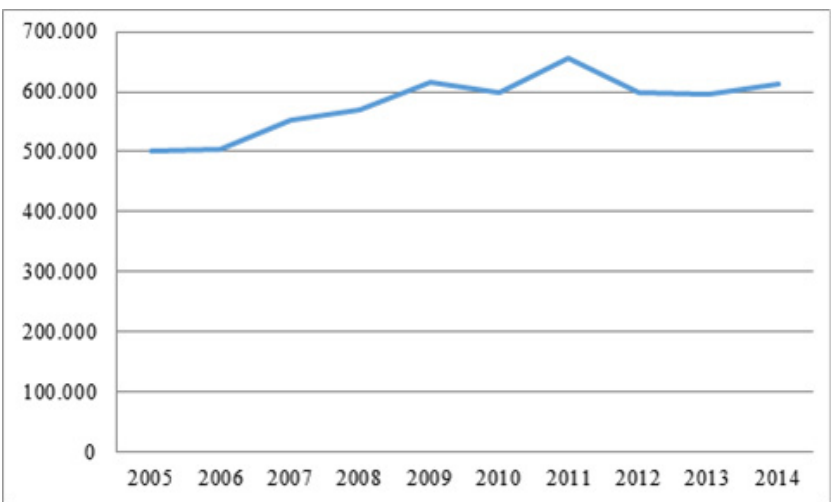

Fuente: Ministerio del Interior (2016).

Otra fuente de información sobre la victimización es la Fundación Paz Ciudadana (FPC) cuyas encuestas periódicas presentan una perspectiva longitudinal en la problemática. Como se puede apreciar en el siguiente gráfico la tendencia por un lapso de tiempo era similar a la encontrada en la Enusc, sin embargo esto cambió en el año 2013 cuando las tendencias cambiaron y se amplió la brecha entre ambas. Se carece de información que permita identificar los motivos que pudieran explicar estas divergencias.

Gráfico 3. Victimización general por hogares Enusc y FPC 2000-2015

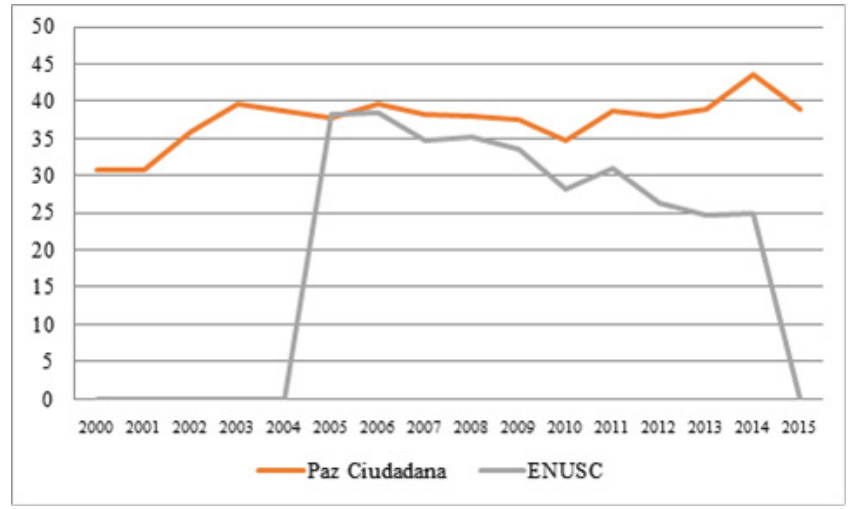

Fuente: Elaboración propia en base a Ministerio del Interior (2016) y Fundación Paz Ciudadana (2016). 
El temor se ha convertido en uno de los problemas de política pública más trascendentes en la última década. Si bien las causas y características de este temor ciudadano están aún en discusión (Gabriel y Greeve 2003), no deja de evidenciarse que un porcentaje importante de la población presenta altos niveles de ansiedad hacia la delincuencia. Al igual que respecto a la victimización, la principal fuente de información fue el Índice de Temor realizado por la FPC. Dicho índice muestra un porcentaje relevante de la población ubicada en los estratos altos de temor. Llamativamente estos porcentajes de la población que expresan tener alto temor no disminuyen en los años donde la victimización habría bajado. Situación que confirma que la inseguridad como percepción se ha consolidado como un problema social autónomo.

Otros indicadores enfatizan en la complejidad de este fenómeno en Chile. Por ejemplo el porcentaje de personas que en las Enusc respondieron que la delincuencia en el país aumentó en los últimos 12 meses alcanzó el 79\% en el 2014. De hecho el periodo 2005-2014 no se evidencian cambios sustanciales. De igual manera casi la mitad de la población afirmó creer que será víctima de un delito en el próximo año en todo el periodo analizado (Ministerio del Interior, 2016).

De esta forma el contexto chileno está marcado por un creciente sentimiento de inseguridad de la población que se encontraría disociado de la tendencia delictual (Muller y Seligson, 1994). La literatura ha mostrado que en otros contextos la percepción de inseguridad va ligada de una crítica a las instituciones gubernamentales y en especial hacia la policía. Este no es el caso de Chile, las características institucionales juegan un rol relevante y se analizará a continuación (Booner, 2013).

\section{La policía chilena}

Carabineros fue fundada en 1927, sigue un modelo de policía principalmente continental, es decir castrense y centralizado, en el artículo $\mathrm{n}$. 1 de la Ley Orgánica Constitucional se define a la institución como:

Carabineros de Chile es una institución policial técnica y de carácter militar, que integra la fuerza pública y existe para dar eficacia al derecho; su finalidad es garantizar y mantener el orden público y la seguridad pública interior en todo el territorio de la República y cumplir las demás funciones que le encomiendan la Constitución y la ley. ${ }^{2}$

\footnotetext{
${ }^{2}$ Ley 18.961. Ley Orgánica Constitucional de Carabineros de Chile.
} 
La institución se autodefine como un como cuerpo armado obediente, no deliberante, profesional, jerarquizado y disciplinado y su personal está sometido a las normas establecidas en la mencionada ley orgánica, su Estatuto, el Código de Justicia Militar y su propia reglamentación interna. Desde su creación, ha realizado múltiples cambios, el más reciente fue pasar su dependencia al Ministerio del Interior en el año 2011, a pesar que estaba entre las definiciones claves de la coalición que ganó las elecciones en el año 1990. Es decir casi dos décadas tomó la realización de una definición política que estaba clara por parte de la coalición de centro izquierda que ha gobernado la mayor parte de este periodo. Las dificultades del proceso de cambio de dependencia institucional son una muestra de los importantes niveles de incidencia que tienen la institución en el debate político.

Desde el regreso de la democracia las instituciones policiales recibieron un fuerte apoyo gubernamental a fin de poder lidiar con uno de los problemas sociales más importante para la ciudadanía (Dammert, 2012). Este respaldo gubernamental estuvo principalmente relacionado con el aumento del presupuesto policial y de la dotación. Con relación al presupuesto nacional, el gasto público en ambas policías ha crecido de forma sostenida y sin mayores debates sobre la utilidad de dicha inversión. De hecho, como se verá posteriormente la principal iniciativa de cambio de la estrategia de patrullaje desarrollada por Carabineros, llamada Plan Cuadrante, ha sido críticamente evaluada por diversas organismos y sin embargo no ha sufrido cambios en sus niveles de crecientes de financiamiento (Hathazy, 2013; Winchester, 2007).

Conocer el financiamiento total de las instituciones policiales es una tarea compleja ya que en los últimos años los gobiernos regionales y las municipalidades han empezado a jugar un rol importante en el financiamiento. Así con el objetivo de responder al reclamo de los vecinos por más seguridad y presencia policial, se convirtieron en fuentes relevantes de financiamiento de la institución, destinado íntegramente a la compra de mayor infraestructura y tecnología. En su informe anual, la Fundación Paz Ciudadana declaró que el gasto público ha aumentado en $106 \%$ en 9 años y alrededor del $62 \%$ se gasta en policías y Ministerio Público (FPC, 2015).

En Chile a fines de los 90s se desarrolló un debate sobre las medidas más eficientes para disminuir la tasa de delitos denunciados. Debido a la reciente emergencia de la problemática, el debate se caracterizó por la falta de precisión y en algunas ocasiones por la carencia de perspectivas informadas adecuadamente. En este sentido, la discusión se planteó en base a discursos ideológicos que establecían la dicotomía entre prevención y control. Las fuerzas políticas de oposición (de centro derecha) propusieron desde un 
inicio la necesidad de políticas de "mano dura" que refuercen el rol policial y endurezcan las penas para los delitos menores, así como se disminuya la edad de imputabilidad. Por otro lado, entró de forma inicial en el debate la relevancia de las medidas preventivas en la disminución de la criminalidad, así como el rol de los medios de comunicación en magnificar la situación.

Desde esta perspectiva, el caso chileno es único. Sin duda en América Latina muy pocos son los países donde las policías se instalan como la institución mejor evaluada del país, además la que tiene más confianza ciudadana. Paralelamente, la sensación de inseguridad como se describió previamente crece pero la ciudadanía no necesariamente culpa a la institución policial del incremento del delito.

\section{La importancia de la confianza ${ }^{3}$}

La confianza es uno de los pilares centrales de la vida en sociedad. Sin ella, se desarrolla el autoritarismo, la fragmentación y la violencia ya que los ciudadanos pierden interés en la relación con sus pares así como en la representación de las instituciones. De esta manera, la confianza se convierte en un tipo de material que une diversos objetivos, valores y necesidades de la sociedad para lograr sus metas comunes. Sin embargo, en la actualidad esa confianza está en una crisis que se puede caracterizar por sus dos facetas: (i) la apreciación sobre el vecindario, la comunidad más directa y la cohesión social. El ambiente social y las expectativas ciudadanas confluyen en la generación de una forma específica de percibir el desorden y de las mejores formas para fortalecer el orden social. La mirada general sobre el "otro" define una manera de entender y tratar de enfrentar la vida en común, ya sea de forma asociativa o basada en el individualismo más evidente. Sin confianza interpersonal es compleja la realización de proyectos comunes, la búsqueda de solución a las limitaciones sociales y la consolidación de una imagen de sociedad integrada. (ii) la confianza institucional revela la magnitud de las fortalezas sociales para consolidar y desarrollar el Estado de Derecho así como las instituciones democráticas. El poder entregado al Estado para la regulación de las actividades sociales requiere de grados de reconocimiento por parte de la población para la realización de las complejas tareas de orden de la vida social.

Las dificultades intrínsecas de la separación entre una y otra son evidentes. En una sociedad donde los ciudadanos no generan lazos de confianza entre ellos, difícilmente se podrá esperar que la confianza institucional sea sólida. ${ }^{3}$ El apartado que sigue también se encuentra, desarrollado más ampliamente, en Dammert
(2014). 
Especialmente reconociendo que las instituciones no son más que agregados de personas que toman decisiones de acuerdo a intereses que deberían ser los aceptados por la mayoría. Analizar el proceso de erosión o fortalecimiento de la confianza requiere de un sistema integral que revise este proceso sinérgico de sumatoria permanente en la que interpersonal e institucionalmente se van consolidando miradas similares en la población.

La confianza de los ciudadanos en las instituciones públicas es un elemento central para el funcionamiento de la democracia. La vinculación entre la población y las instituciones que deben representarla necesita de confianza como pilar fundante para establecer la legitimidad de sus actuaciones.

Múltiples son los estudios que han analizado los procesos de democratización en América Latina tomando en cuenta esta variable (Pnud, 2002; Borja, 2003). Basados en la necesaria legitimidad del sistema (Power, 2002), la confianza en las instituciones (Arriagada y Godoy, 1999) y el capital social presente en cada contexto. La literatura explicita el impacto negativo que tiene la criminalidad sobre la confianza en el gobierno (Chanley, Rudolph y Rahn, 2000; Vlassis, 2000). Es decir, la evidencia empírica muestra que la percepción de aumento de la inseguridad asociada a la delincuencia hace disminuir la confianza en las instituciones políticas en general y, especialmente, en las de control social formal como el sistema de justicia y la policía. Ahora bien, la desconfianza en las instituciones policiales y judiciales no sólo se vinculan con la falta de efectividad en las tareas que realizan sino también con la percepción de impunidad y arbitrariedad de sus labores. La percepción generalizada que la justicia no se imparte por igual para todos así como los abusos en el accionar policial tiene un impacto clave en la confianza institucional.

La metáfora de la puerta giratoria es una demostración de esta extendida sensación de impunidad que tienen los ciudadanos. Así entendida, los castigos no existen o no son los adecuados para aquellos que cometen algún delito. Es notable que en Chile los niveles de encarcelamiento han crecido sustancialmente, las leyes han endurecido los castigos y se ha bajado la edad de imputabilidad penal, pero nada de eso ha sido suficiente para disminuir la percepción de impunidad en el país. En este proceso los medios de comunicación juegan un rol relevante ya que cotidianamente presentan hechos cometidos por infractores reiterados o magnifican la información sobre bajas condenas o libertades provisionales.

\section{Inseguridad, desconfianza y autoritarismo}

Chile vivió bajo dictadura militar por 17 años (1973-1990) marcada por la represión política, la permanente violación de derechos humanos y 
la persecución de todos aquellos considerados enemigos del sistema. La dictadura de Pinochet ha dejado innegables marcas en la sociedad chilena que van desde la forma como se organiza el estado, la constitución hasta el modelo económico. Adicionalmente, se debe destacar que, contrario a lo que ocurrió en Argentina, el apoyo al sistema fue sostenido en el tiempo. Es así como en el plebiscito de 1989 y las elecciones presidenciales de 1990 aquellos que apoyaron al régimen superó el 45 por ciento.

En teoría el temor está muy fuertemente vinculado con la confianza/ desconfianza en la capacidad de las instituciones del control social formal de ejercer eficazmente su rol. Así, mientras mayor sea la confianza en las policías y sistema de justicia menor será la inseguridad o sensación de temor de las personas y, viceversa, cuando la relación de la población con estas instituciones esté marcada por la desconfianza el temor de los ciudadanos será mayor. Pero la situación en Chile contradice esta hipótesis y por ende desafía aquellos análisis basados en esta perspectiva.

La perspectiva del análisis institucionalista, la valoración que la población tiene del trabajo que realizan los policías y operadores del sistema judicial (jueces, fiscales, ministros de cortes, abogados y funcionarios) tendrá efecto en la confianza en las instituciones que ellos representan. En cambio, si se asume el enfoque culturalista, la confianza en las instituciones debiera estar fuertemente respaldada por prácticas de confianza y cooperación entre la población. Encontrar elementos que comprueben una u otra hipótesis tendrá efectos prácticos en el diseño de políticas. La falta de confianza en la policía y la justicia y, por lo tanto, la sensación de indefensión frente a la amenaza delincuencial, puede llevar a las personas a desarrollar un discurso autoritario alternativo al ejercicio legítimo de autoridad, como único recurso frente a un peligro que consideran inminente y cuyo extremo es la justicia por mano propia.

\section{La confianza en Carabineros}

Chile es uno de los pocos países latinoamericanos donde la confianza en las policías, pero especialmente en la policía uniformada supera a todas las instituciones gubernamentales, incluso la iglesia y los medios de comunicación. Como se ha identificado previamente la confianza en las instituciones es un arma poderosa para legitimar su actuación frente a los ciudadanos pero también para avanzar con mayor fuerza en niveles de autonomía frente al poder político que está mucho menos valorado por la ciudadanía. La paradoja no es de simple resolución, en Chile la ciudadanía siente temor y considera que los problemas 
de seguridad no se solucionan pero culpa principalmente al gobierno y a la justicia por esta situación.

Si bien se esperaría que la mala evaluación de la situación y las iniciativas de seguridad impacten sobre la valoración de las policías o su percepción de desempeño, la información disponible muestra un fenómeno distinto. Por un lado la ciudadanía reconoce que las policías están "de manos atadas" por qué no tienen las funciones o capacidades necesarias para actuar en situaciones delictivas. Por otro, las policías serían las víctimas de una "puerta giratoria" instalada en el sistema de justicia que deja que los detenidos salgan libres sin mayores castigos. Estas dos imágenes ciudadanas están muy arraigadas en el discurso cotidiano en Chile y si bien no se evidencia la presencia de análisis que expliciten el tránsito de esta narrativa, se puede afirmar que los medios de comunicación juegan un rol innegable (Bonner, 2013).

De esta forma como se puede apreciar en el siguiente gráfico el porcentaje de población que dice tener mucha o bastante confianza en Carabineros de Chile en la última década ha estado por encima del 40\%. Guarismo muy superior al que se encuentra en muchas otras instituciones públicas. Este hecho se ha convertido en pieza angular de las comunicación que interna y externamente desarrolla la institución centrada no sólo en evidenciar sus logros sino también en el apoyo ciudadano.

Gráfico 4. Confianza en Carabineros de Chile

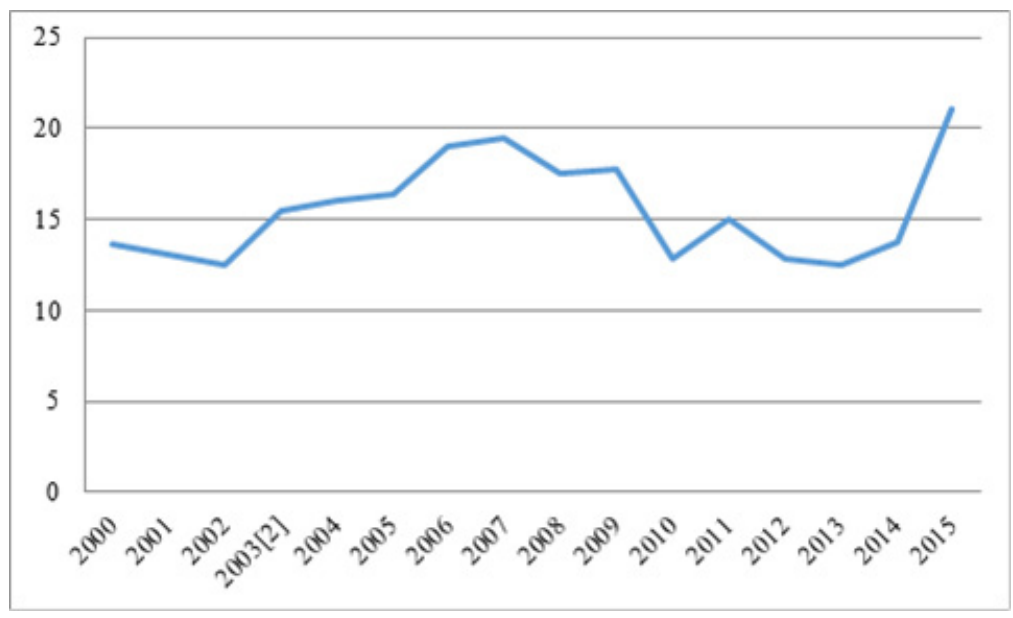

Fuente: Fundación Paz Ciudadana (2016) 
Otra encuesta de opinión desarrollada por la Fundación Paz Ciudadana ha relevado la calificación que los ciudadanos otorgan a las principales instituciones vinculadas con la justicia criminal. ${ }^{4}$ Los resultados confirman que la institución policial cuenta con resultados positivos durante todo el periodo de implementación de la encuesta. Vale recordar que la pregunta es calificación del 1 al 7 (forma que se utiliza en Chile para calificar en el sistema educativo) donde a partir del 4 se estaría aprobando en términos de evaluación total. El gráfico siguiente muestra que en los últimos 15 años la institución policial ha recibido notas aprobatorias en todo el periodo muy por encima del Ministerio Público y los jueces que han sido reprobados sistemáticamente.

Gráfico 5. Aprobación del desempeño institucional 2000-2015

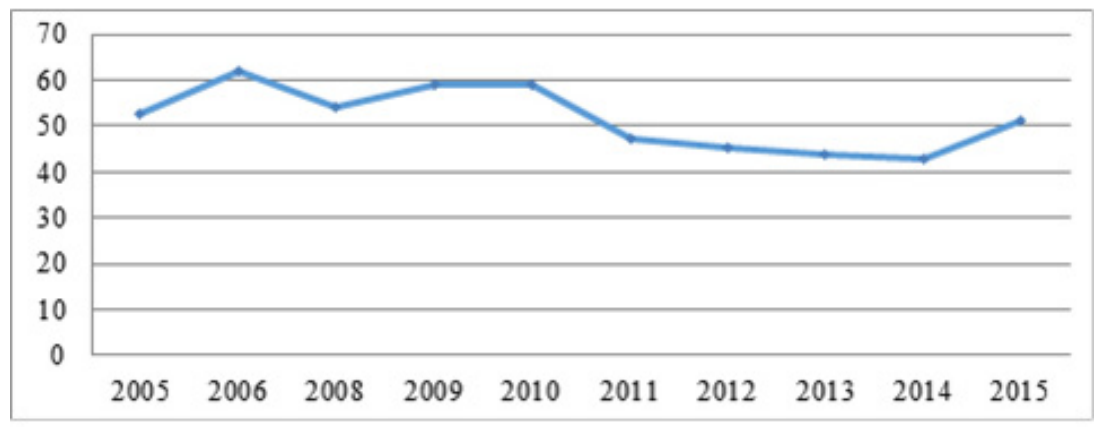

Fuente: Elaboración propia en base a Encuesta UDP (2016)

La importancia ciudadana de la labor de Carabineros se ha visto traducida en un aumento sostenido de su dotación y presupuesto. De hecho, el actual gobierno de la presidenta Bachelet (2012-2016) incluyó en su propuesta de gobierno el aumento de 6 mil plazas para Carabineros de Chile así como un aumento permanente del presupuesto destinado a la institución. Este compromiso de aumento de personal y presupuestario con la institución ha sido una constante que todos los gobiernos han replicado desde el regreso a la democracia.

La evaluación promedio de los jueces, por otro lado, ha sido negativa durante todo el periodo y ha mostrado una tendencia decreciente en los últimos años. Cabe destacar que el discurso político ha hecho énfasis en las dificultades

\footnotetext{
${ }^{4}$ Para mayor detalle ver $<$ http://www.pazciudadana.cl $>$.
} 
que pondrían los jueces para castigar a los imputados. ${ }^{5}$ Debate especialmente llamativo en un país que tiene una de las tasas más altas de encarcelamiento de América Latina. Finalmente, el Ministerio Público ha tenido un rol complejo que jugar desde su creación dada la percepción ciudadana de poca efectividad de su labor. Esta sensación ha ido acompañada de un constante debate público sobre la limitada capacidad de los fiscales para efectivamente castigar a los imputados y resolver los casos que investigan. ${ }^{6}$ En su mayoría los debates se han desarrollado en los medios de comunicación, es decir no están basados en información o estudios sino más bien en enfrentamientos de corte político que tiene por objetivo disminuir la percepción que el gobierno de turno no cumple con las tareas de control y prevención del delito.

Una de las principales políticas públicas implementadas desde el regreso de la democracia ha sido la reforma del proceso penal que incluyó la creación del Ministerio Público y la Defensoría Penal Pública (Muñoz, 2011). Pensado como un proceso reformador que traería mayor celeridad y justicia a los procesos penales así como disminuiría el tiempo de tramitación de las causas, la reforma enfrentó diversas adecuaciones en los primera década de implementación. Un porcentaje importante de estos cambios se vincularon con devolver capacidades a los funcionarios policiales para que realicen labores de investigación sin la intervención inicial de un fiscal. La llamada reforma de la reforma (Vargas, 2011) se vinculó también con la percepción que los delitos habían aumentado principalmente por que se restringieron las capacidades policiales para controlar el delito.

Pero los promedios no representan la realidad con sus diversas aristas. El análisis más detallado de la información para el año 2010 evidenció que la relación entre confianza y nivel socioeconómico es directamente proporcional, es decir a mayor nivel socioeconómico se presentan mayores niveles de confianza en Carabineros. Es decir aquellos que pertenecen a los sectores menos privilegiados, y por ende requieren más del trabajo policial por la ausencia de seguridad privada y mayores niveles de precariedad, tienen una relación más compleja con la institucionalidad policial. Así el porcentaje de

\footnotetext{
${ }_{5}$ Alguna de la cobertura de medios sobre la temática: < biobiochile.cl/noticias/2015/07/30/ presidente-de-asociacion-de-magistrados-de-chile-es-irresponsable-culpar-a-jueces-pordelincuencia.shtml>; <lanacion.cl/juica-hay-cierta-inseguridad-publica-pero-no-la-hanprovocado-los-jueces-y-fiscales/noticias/2011-11-11/164650.html>; <latercera.com/noticia/ opinion/ideas-y-debates/2012/10/895-488065-9-leyes-y-jueces-garantistas.shtml>.

${ }_{6}<$ lasegunda.com/Noticias/Nacional/2012/10/788249/jueces-garantistas-en-la-mira-del -gobierno-jovenes-empoderados-y-desafiantes>; <lasegunda.com/Noticias/Nacional/2011/ 11/697470/nueva-arremetida-de-hinzpeter-contra-fiscales-ahora-es-por-detencion-en-caso-dedrogas>.
} 
población de alto nivel socioeconómico que declaró confiar poco o nada en Carabineros alcanzó el 30\% mientras que en los sectores populares superó el $42 \%$. De igual forma, la confianza institucional tiene un claro sesgo etario que se confirma cuando casi el $44 \%$ de los jóvenes entre 18 y 29 explicitó no confiar poco o nada en Carabineros mientras que este porcentaje disminuye a $29 \%$ en los mayores de 61 años (UDP, 2016).

Esta desigual distribución de la confianza ciudadana con Carabineros no solo se organiza por los grupos definidos previamente. En una reciente encuesta realizada por la FPC se establecieron los niveles de confianza de acuerdo a un eje de cercanía y conocimiento del trabajo policial. El siguiente gráfico presenta los resultados para el periodo 2010-2015 de aquellos que presentaron mucha y bastante confianza en Carabineros. Como se puede observar, los que tienen una mayor cercanía con el trabajo policial, es decir aquellos que han sido víctimas de un hecho delictivo y adicionalmente denunciaron el hecho, son los que menor nivel de confianza tienen en la institución policial. Finalmente los que mayor nivel de confianza tienen en la institución son aquellos que no han sido víctimas de delito en el último año.

Gráfico 6. Confianza en Carabineros, 2010-2015

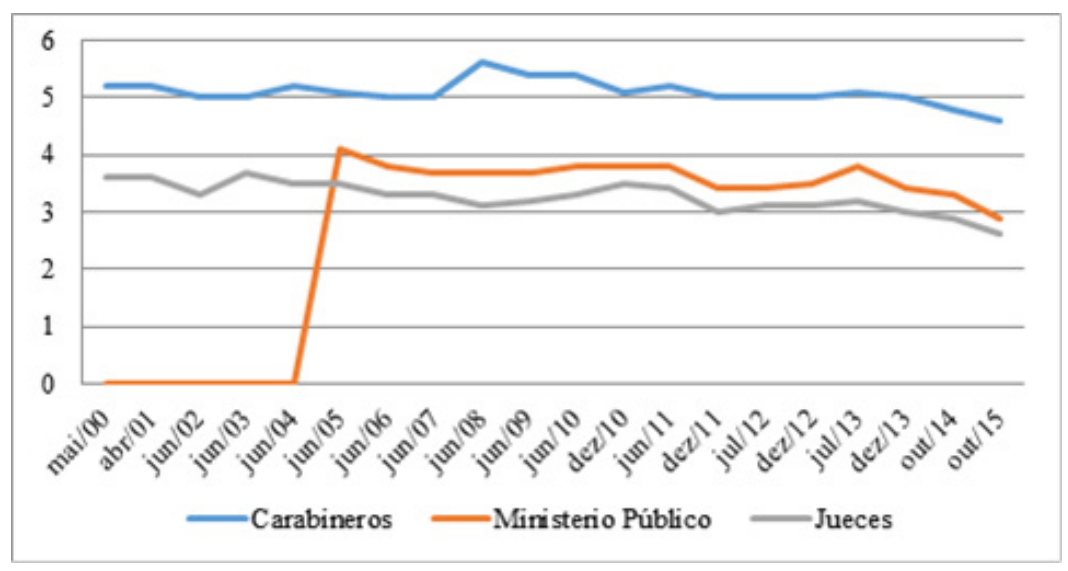

Fuente: Elaboración propia en base a Fundación Paz Ciudadana (2016).

La limitada información disponible empieza a describir un panorama que requiere de cambios relevantes para que aquellos que más requieren del servicio policial mejoren la percepción de su accionar. Para de esta forma evitar la posible paradoja que el mejor servicio policial se esté entregando a aquellos 
que menos lo necesitan. Confirmar esta hipótesis requiere de investigación cualitativa que se concentré en aquellas áreas o grupos poblacionales que enfrentan mayores niveles de inseguridad.

La literatura disponible no permite avanzar en la confirmación de las hipótesis propuestas previamente, pero permiten confirmar que se requieren de procesos de modernización y reforma de las prácticas policiales en Chile. Lamentablemente, la misma confianza ciudadana puede convertirse en un elemento que limite los procesos de modernización necesarios para avanzar con una efectiva policía democrática con importantes niveles de autonomía profesional pero subordinado a las decisiones del poder político.

Gráfico 7. Confianza en Carabineros, 2010-2015

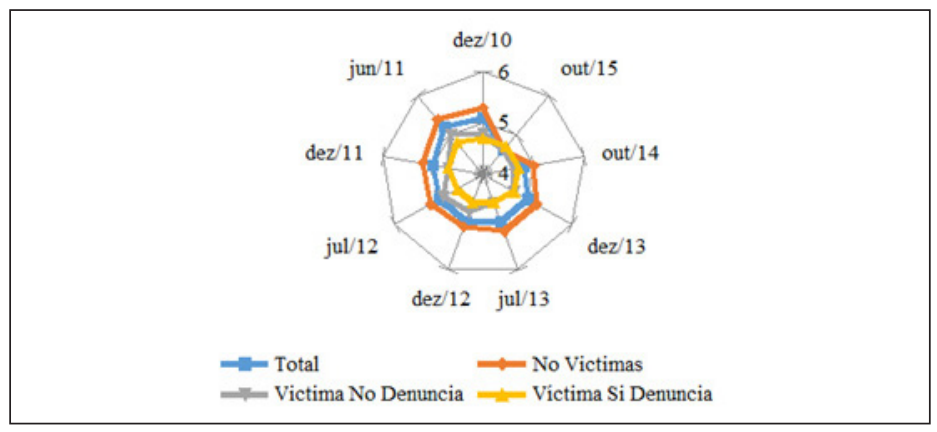

Fuente: Fundación Paz Ciudadana (2016).

\section{Reformas policiales en democracia}

Desde el regreso a la democracia en Chile los cambios desarrollados en Carabineros de Chile han sido impulsados, generados y desarrollados desde el interior de la institución. Son pocas las instancias o reformas generadas desde la sociedad civil, los expertos o las propuestas de gobierno dado que los espacios de participación de estos actores en los temas policiales son mínimos. La Constitución que rige Chile que dada del régimen militar de Pinochet, dejó múltiples instancias de salvaguarda que no permiten reformar instancias educativas, de distribución del personal e incluso de formato de evaluación del ingreso de los postulantes. Pero si esos candados fueron dejados por la dictadura, los partidos políticos en democracia no hicieron mucho para cambiarlos. En temas sustanciales como la distribución policial por ejemplo todavía pesa un fuerte sesgo informativo que reserva la información a prácticamente cualquiera fuera de la institución que quiera saber cuáles son 
los números exactos de distribución policial en el territorio. Las quejas de los alcaldes no han sido pocas cuando afirman no tener suficiente dotación o no tener capacidad real de definición sobre sus prioridades. De igual forma, la reforma de la justicia militar es un tema con más de dos décadas de espera que requiere también de una reforma constitucional. En la actualidad uno de los puntos que más controversia genera es la posibilidad que tienen las cortes castrenses de juzgar a funcionarios de Carabineros cuando se han visto involucrados en delitos en que civiles han resultado con lesiones o fallecidos.

Este tema ha estado en prácticamente todos los planes de gobierno de la centro izquierda chilena y sin embargo llegado al poder no han podido (o no han querido) avanzar en su implementación. ${ }^{7}$ El gobierno de Sebastian Piñera (2010-2014) introdujo una modificación legal que se hizo cargo de esta situación, excluyendo el juzgamiento de civiles por parte de la justicia militar en la ley 20.477 del 30 de diciembre de 2010. Pero los cambios sustantivos siguen esperando. Adicionalmente, en los últimos años se han aprobado diversos cuerpos legales que han aumentado las penas de aquellos que atenten contra funcionarios de carabineros dejando explícito que las tentativas se castigarán como hechos consumados. ${ }^{8}$

A nivel estructural, por ejemplo, la creación del departamento de análisis criminal en noviembre del 2011 encargado del estudio y análisis de variables criminológicas del delito orientado a satisfacer la necesidad de información estratégica y operativa. ${ }^{9}$ Se consolidó internamente antes de tener alguna vinculación clara con las instancias gubernamentales encargadas precisamente de las mismas tareas en el Ministerio del Interior y Seguridad Pública.

Otra área donde los cambios se han generado desde adentro es la estrategia de trabajo policial, especialmente vinculado con el Plan Cuadrante considerado el principal esquema de trabajo policial de la última década. El Plan Cuadrante nació al interior de la institución con un cambio de los indicadores de gestión, de evaluación y especialmente de distribucíon de la dotación policial. Mientras en otras policías de la región, debido a escándalos de corrupción, uso de la fuerza o general percepción de ineficiencia se generaban programas de policía comunitaria para enfrentar el incremento de la inseguridad; en Chile Carabineros formulaba una propuesta de redefinición interna de la que el gobierno se enteró posiblemente cuando ya no quedaban más que algunos detalles de definición.

\footnotetext{
$7<$ radio.uchile.cl/2015/06/12/reforma-a-la-justicia-militar-uno-de-los-pendientes-del-gobierno>.

$8<$ camara.cl/sala/verComunicacion. aspx ? comuid $=14412 \&$ formato $=$ pdf $>$.

${ }^{9}$ Orden general de Carabineros n. 002272 del 6 de junio del 2014.
} 
Las evaluaciones iniciales del plan cuadrante evidenciaron múltiples signos de limitaciones y necesidades de cambios, pero no hay sido tomadas en cuenta. Así por ejemplo, en la evaluación realizada por Fundación Paz Ciudadana se concluyó:

No se distingue en las comunas con Plan Cuadrante una gestión de oferta que se acople a la demanda - según la lógica de elasticidad de oferta -, que sea constante o tenga una tendencia al alza y que sea significativamente mayor respecto a lo que ocurre en igual parámetro en unidades sin Plan Cuadrante. Por lo tanto, no se puede evidenciar una gestión de recursos diferenciada en unidades con Plan Cuadrante de Seguridad Preventiva, en relación a aquellas unidades que no trabajan bajo esta metodología. Esto apoya la hipótesis n. 1 que señala que la presencia de recursos sería más relevante que el modelo de gestión que implica el Plan Cuadrante en las comunas (FPC, 2014).

Adicionalmente concluyó que "de los 117 cuarteles estudiados, el 85\% de las reducciones de oferta se explican por una disminución del recurso humano. Si se considera que esto se da en un contexto de una demanda creciente, entonces puede reflejar una gestión de recurso humano ineficiente" (FPC, 2014). Esta evaluación no estuvo disponible por mucho tiempo y solo recientemente fue publicada por la FPC en su página web, Carabineros respondió objetando la metodología del estudio. Otra evaluación estableció “el diseño actual del Programa no presenta acciones destinadas hacia un enfoque de policía comunitaria (enfoque que inspiró la creación del programa) y el diseño general ha reforzado básicamente una mayor dotación de recursos policiales en aquellas comunas que participan del Plan Cuadrante. Luego de esta evaluación no se generaron disminuciones evidentes en el presupuesto destinado al programa, por el contrario se consolidó una línea de inversión que continuó con el programa e incluso lo potenció. La respuesta institucional ha sido avanzar con espacios cerrados de debate y mejoramiento de la oferta institucional. De hecho se firmó un contrato con una universidad privada para que realice las evaluaciones de los programas e iniciativas institucionales las que tampoco son de dominio público. ${ }^{10}$

Posiblemente la situación descrita previamente no procedería con prácticamente ninguna otra institución del gobierno chileno. Si bien los candados de protección que la dictadura dejó instalado para Carabineros en la Constitución son amplios, la consolidación de una mirada positiva por

\footnotetext{
${ }^{10}<$ ing.uc.cl/uc-entrega-proyecto-de-mejoramiento-del-plan-cuadrante-carabineros/>.
} 
parte de la ciudadanía ha sido el principal barrera para avanzar con medidas que permitan corregir los errores con una mayor intervención de los actores políticos.

Carabineros no se puede comparar con ninguna policía de América Latina. Por décadas ha consolidado un fuerte, profesional, educado y limitado grupo de oficiales que gobiernan la institución y sus procesos. También se ha consolidado un equipo civil asimilado a la institución que apoya la administración de la salud, la previsión y las múltiples tareas de construcción de recintos policiales, compra de materiales, etc. que se concentran de forma ordenada y jerárquica. Los hechos de corrupción no son cotidianos, por el contrario, aquellos hechos conocidos generalmente reciben una respuesta rápida con una desvinculación de la institución por parte de aquellos que son encontrados vinculados con los hechos. Ahora bien, que no sean cotidianos no significa que en los últimos años no se hallan evidenciado diversos espacios de corrupción de variada índole dentro del trabajo policial. Seguramente estas situaciones han generado cambios desde el interior de la institución porque ni el ejecutivo ni el legislativo han dado señal alguna de preocupación por la materia. El mismo derrotero tienen los temas de posible uso excesivo de la fuerza, con una diferencia, la institución muy pocas veces tiene respuestas de exoneración o alejamiento de la institución para los involucrados (Fuentes, 2005).

La confianza en Carabineros se convierte así en una espada de doble filo, que sustenta y sostiene la legitimidad del trabajo policial por un lado pero también inhibe el accionar de los actores políticos y sociales para enfrentar los procesos naturales de cambio y reforma institucional. Más investigación es necesaria para avanzar en lo que podría ser un concepto innovador y poco utilizado de confianza negativa que al igual que el capital social negativo tiene aspectos inicialmente valorables pero que una vez pasado un determinado nivel se puede convertir en una característica que limite las necesarias reformas institucionales.

\section{Referencias}

ARRIAGADA, Irma; GODOY, Lorena. Seguridad ciudadana y violencia en América Latina: diagnóstico y políticas en los años noventa. Santiago de Chile: Cepal, 1999. Serie Políticas Sociales.

BONNER, Michelle. The politics of police image in Chile. Journal of Latin American Studies, v. 45, n. 4, p. 669-694, $2013<10.1017 /$ S0022216X13001168>.

BORJA, Jordi. La ciudad conquistada. Madrid: Alianza Editorial, 2003. 
CENTRO de Estudios Publicos. Estudio nacional de opinión pública. Encuesta CEP, n. 77, 2016 <cepchile.cl/cep/site/artic/20160818/asocfile/20160818165239/ encuestacep_julij_agosto2016.pdf> (7 nov. 2016).

CHANLEY, Virginia; RUDOLPH, Thomas; RAHN, Wendy. The origins and consequences of public trust in government: a time series analysis. Public Opinion Quarterly, v. 64, n. 3, p. 239-256, $2000<10.1086 / 317987>$.

DAMMERT, Lucia; Mary, MALONE. Fear of crime or fear of life? Public insecurities in Chile. Bulletin of Latin America Research, v. 22, n. 1, p. 79-101, $2003<10.1111 / 1470$ 9856.00065>.

DAMMERT, Lucia. Fear and crime in Latin America: redefining state-society relations. New York: Routledge, 2012.

DAMMERT, Lucía. La relación entre confianza e inseguridad: el caso de Chile. Revista Criminalidad, v. 56, n. 1, p. 189-207, 2014.

FUENTES, Claudio. Contesting the iron fist: advocacy networks and police violence in democratic Argentina and Chile. New York: Routledge, 2005.

FUNDACION Paz Ciudadana (FPC). Evaluación del plan cuadrante de carabineros de Chile, 2014 <www.pazciudadana.cl/wp-content/uploads/2014/07/informe-final-n4-evaluacion-pcsp-2012-fpc.pdf> (4 dic. 2016).

FUNDACION Paz Ciudadana (FPC). Balance de la delincuencia en Chile 2014: radiografía del sistema de seguridad y justicia. Santiago de Chile: FPC, $2015<$ www. pazciudadana.cl/wp-content/uploads/2015/08/balance-de-la-delincuencia-en-chilefpc-17-agosto-2015.pdf> (7 nov. 2016).

GABRIEL, Ute; GREEVE, Werner. The psychology of fear of crime: conceptual and methodological perspectives. British Journal of Criminology, v. 43, n. 3, p. 600-614, $2003<10.1093 / \mathrm{bjc} / \mathrm{azg} 600>$.

HATHAZY, Paul. Fighting for a democratic police: politics, experts and bureaucrats in the transformation of the police in post-authoritarian Chile and Argentina. Comparative Sociology, v. 12, n. 4, p. 505-547, $2013<10.1163 / 15691330-12341271>$.

LECHNER, Norbert. Desafios de un desarrollo humano: individualización y capital social. Contribución al Foro Desarrollo y Cultura organizado para Science Po para la Asamblea General del Banco Interamericano de Desarrollo, BID, 1999.

LEON, Tomás; GREZ, Marcela; PRATO, Juan Andrés; TORRES, Rafael; RUIZ, Sergio. Violencia intrafamiliar en Chile y su impacto en la salud: una revisión sistemática. Rev. méd. Chile. v. 142, n. 8, p. 1014-1022, $2014<10.4067 /$ S003498872014000800009>.

MINISTERIO del Interior y Seguridad Publica de Chile. Encuesta nacional de victimización y seguridad ciudadana, 2016 < seguridadpublica.gov.cl/encuestas/>.

MULLER, Edward; SELIGSON, Mitchell. Civic culture and democracy: the question of causal relationships. American Politic Science Review, v. 88, n. 3, p. 635-652, 1994 $<10.2307 / 2944800>$. 
MUÑOZ, Daniel. La reforma procesal penal en Chile: nuevos agentes, sus trayectorias y la reestructuración de un campo. Revista Política, v. 49, n. 1, p. 43-70, 2011 $<10.5354 / 0716-1077.2011 .16309>$.

PLISCOFF, Cristian. Police reform in times of collaboration. Public Administration Review, v. 69, n. 1, p. 151-153, $2008<10.1111 /$ j.1540-6210.2008.01956.x>.

PNUD. Informe de desarrollo humano: nosotros los chilenos - un desafío cultural. Santiago de Chile: Pnud, 2002.

POWER, Timothy. La confianza interpersonal brasileña en perspectiva comparada. América Latina Hoy, n. 32, p. 91-115, 2002.

RAMOS, Marcela; GUZMAN, Juan. La guerra y la paz ciudadana. Santiago de Chile: LOM, 2000.

UDP [Universidad Diego Portales]. Encuesta Nacional UDP 2015. Santiago, 2016 $<$ http://encuesta.udp.cl/>.

VARGAS, Juan Enrique. Estado actual de la reforma procesal en Chile. Anuario de Derechos Humanos, v. 2, p. 185-188, $2006<10.5354 / 0718-2058.2006 .13385>$.

VLASSIS, Dimitri. Long arm of the law. The World Today, v. 56, n. 2, p. 10-11, 2000.

WINCHESTER, Lucy (org.). Informe final de evaluación: programa lan cuadrante de seguridad preventiva. Chile: Ministerio de Defensa, subsecretaría de Carabineros, 2007 <dipres.gob.cl/574/articles-140457_informe_final.pdf> (7 nov. 2016).

Autora correspondiente:

Lucia Dammert

Lo Beltrán 2471 Vitacura

Santiago, Chile

Recibido el: 19 jun. 2016.

Aprobado el: 7 nov. 2016. 